\title{
Locating the Shadow Regions in LiDAR Data: Results on the SHARE 2012 Dataset
}

\author{
Mustafa BOYACI, Seniha Esen YUKSEL* \\ Hacettepe University, Department of Electrical and Electronics Engineering \\ Beytepe, Ankara, Turkey \\ Contact: mboyaci@aselsan.com.tr, eyuksel@ee.hacettepe.edu.tr
}

\begin{abstract}
In hyperspectral imaging, shadowy areas present a major problem as targets in shadow show decreased or no spectral signatures. One way to mitigate this problem is by the fusion of hyperspectral data with LiDAR data; since LiDAR data presents excellent information by providing elevation information, which can then be used to identify the regions of shadow. Although there is a lot of work to detect the shadowy areas, many are restricted to distinct platforms like ARGCIS, ENVI etc.

The purpose of this study is to (i) detect the shadow areas and to (ii) give a shadowiness scale in LiDAR data with Matlab in an efficient way. For this work, we designed our Line of Sight (LoS) algorithm that is optimized to run in a Matlab interface. The LoS algorithm uses the sun angles (altitude and azimuth) and elevation of the earth; and marks the pixel as "in shadow" if there lies an object of higher elevation between a given pixel and the sun. This is computed for all pixels in the scene and a shadow map is generated. Further, if a pixel is marked as a shadow area, the algorithm assigns a different darkness level which is inversely proportional to the distance between the current pixel and the object that causes the shadow. With this shadow scale, it is both visually and computationally possible to distinguish the soft shadows from the dark shadows; an important information for hyperspectral imagery. The algorithm has been tested on the SHARE 2012 Avon AM dataset. We also show the effect of the shadowiness scale on the spectral signatures.
\end{abstract}

Keywords: LiDAR, hyperspectral, shadow area, line of sight.

\section{INTRODUCTION}

Remote sensing technology uses propagated signals to get information about an object or a wide field without any physical contact, which makes the information reachable for dangerous or inaccessible areas. Remote sensing technology can be classified into the active and passive technologies. Hyperspectral imaging, a passive remote sensing technology, collects sun beams which are reflected from the Earth's surface. LiDAR, on the other hand, is an active remote sensing technology that transmits optical laser lights in pulses and determines the height of the target by using speed of light. LiDAR includes elevation, coordinate and intensity of laser beam information by calculating speed of light and the difference between emission and collection precise times of laser beams and using an internal GPS system.

In hyperspectral imaging, the measured target reflectance data is used in material detection or classification by using that all materials have different hyperspectral signatures for an interval of some common wavelengths. Shadowy areas and different objects that are made of the same raw material present significant problems in detection. For example, paved roads and roofs have similar reflectances. In fact, the main difference between paved roads and roofs, is their heights which hyperspectral data doesn't include. Also, shadowy areas have lower reflectance values compared to the areas where the sun lights directly reach. LiDAR data can be used as auxiliary data to hyperspectral data to defeat both these major problems ${ }^{1,2,3}$.

Shadowy areas are determined by using aerial LiDAR data and line of sight algorithm ${ }^{4,5}$. The line of sight is an assumed line from target to the light source which is the Sun. If this line is interrupted by a higher object, the target is classified as shadow. Shadowy areas are marked in hyperspectral data with the use of LoS algorithm. 
This study could be separated from other LoS algorithms that using MATLAB interface and calculating a darkness level. The LoS algorithm uses parameters such that altitude and azimuth angles of the sun at the certain date and time instances in which hyperspectral data was collected, since shadow regions are used on hyperspectral data.

\section{THE SHARE 2012 DATASET}

\subsection{Dataset}

A multi-modal imaging data was collected over Avon, Rochester (only hyperspectral data), Conesus Lake, Hemlock Lake and a nearby quarry, which is called Share 2012 Dataset, with hyperspectral, multispectral and LiDAR sensors to support future research and provide shareable resource ${ }^{6}$.

Avon dataset was collected on September 20, 2012, by airborne sensors both in the morning and the afternoon. In this study, Avon dataset, which was collected in morning, was used for testing the developed LoS algorithm.

\subsubsection{LiDAR Dataset}

Leica ALS60 LiDAR system was used to collect LiDAR dataset which is a scanning mirror LiDAR mapping system by Kucera International. This sensor system operates with a $1064 \mathrm{~nm}$ laser and has in inertial navigation system which is Leica IPAS to save and compress collected data in the common LiDAR Data Exchange Format (*.LAS files) ${ }^{6,7}$.

Avon A.M. LiDAR dataset is classified by flight lines and tiles (Figure 1). In the tile data, regions are investigated individually. Therefore, tile data may not be the proper one to use for the LoS algorithm since higher altitude obstacles from nearby tiles might create elusive shadow for individual tiles.

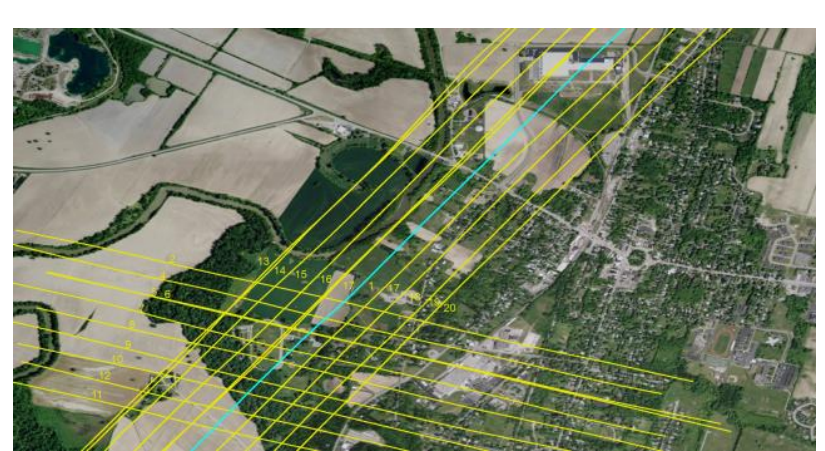

(a)

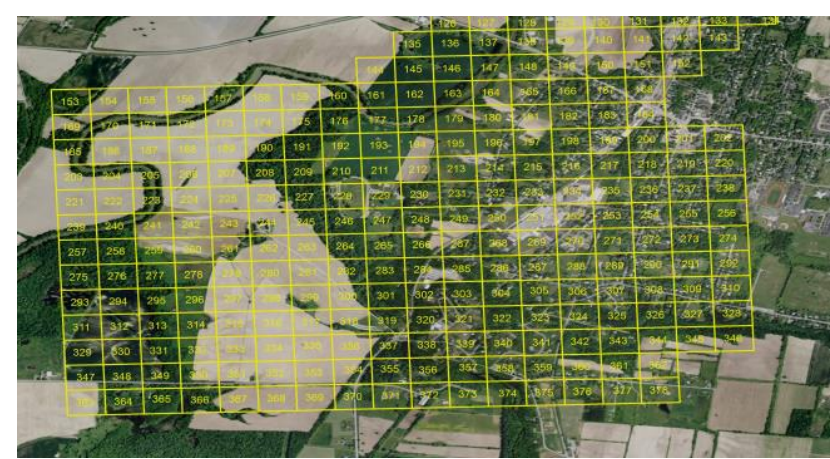

(b)

Figure 1. Morning WASP/LiDAR dataset for the Avon ground truth site that is classified by flight lines in (a) and tiles in (b). In this study, flight line number 16 dataset from (a) was used.

In this study, flight line number 16 dataset was used as shown in Fig.1, which is placed corrected by flight lines, to overcome the above problem and to achieve maximum intersection area with hyperspectral dataset. This data includes a $3554 \times 2903$ elevation matrix, cell size value and UTM (Universal Transverse Mercator) coordinates. Cell size is a distance parameter in meters that is between two neighbor sample points and its value is 1.278 meters for sample data.

\subsubsection{Hyperspectral Dataset}

Hyperspectral imagery data was collected and processed by ProSpecTIR-VS sensor. The spectral range of this sensor is between $400 \mathrm{~nm}$ and $2450 \mathrm{~nm}$ with 360 bands. Also, this sensor system has an internal GPS that is Novatel SPAN-CPT. The software package ATCOR4 utilizes standard MODTRAN4 radiative transfer code to convert the calibrated radiance data to surface reflectance value. After the atmospheric absorption and scattering components, ATCOR4 generates log files for all flight lines that include program settings and input parameters ${ }^{8}$. Position data of the image is collected by Inertial Navigation System in UTM coordinates which is boresighted in accordance with hyperspectral sensor $6,8,9$

On Avon site, there were six flights on September 20, 2012, three of which were not proper to use due to VNIR saturation on buildings, low exposure and high exposure problems ${ }^{8}$. In this study, flight line number 1 , which is called 
0920-1638, was used with LiDAR dataset. Elevation of this flight was 936 meters and data was collected for 320 samples and 5873 lines $(5873$ x 320 matrix $)$ for 360 bands. At the flight time and date, the zenith angle of the sun was 46.4 degrees and the azimuth angle of the sun with respect to north was 149.6 degrees. The altitude angle of the sun is equal to '90 degrees - zenith angle' (Figure 3).

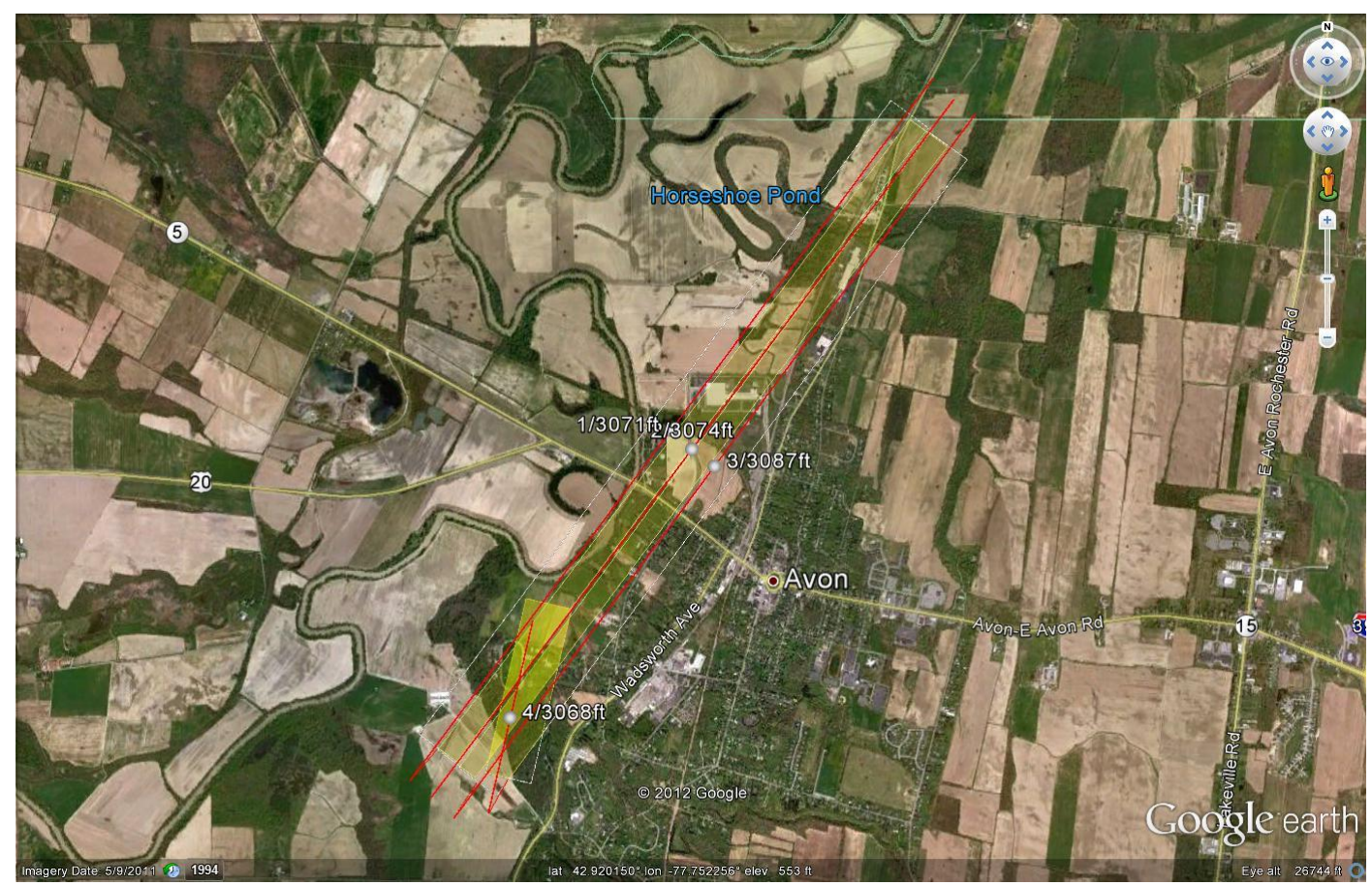

Figure 2. Morning ProSpecTIR-VS flight lines for Avon site.

\section{METHODOLOGY AND EXPERIMENTAL RESULTS}

\section{Line of Sight Method}

In the line of sight method, a point is marked as shadow where the sun light cannot reach directly. The altitude (or zenith), azimuth angles of the sun and the heights of the local objects are used in classifying the point as in shadow or not as shown in Figure 3. The angles of the sun, as shown in Fig. 3(a), were obtained from the header file of the hyperspectral data file. The zenith angle is 46.4 degrees and the azimuth angle is 149.6 degrees. In Fig 3(b), there is a high object which causes the shadow. If the high object intersects the line of sight of the target point due to its length, the target point is classified as shadow and a shadowiness level is assigned to this point. 


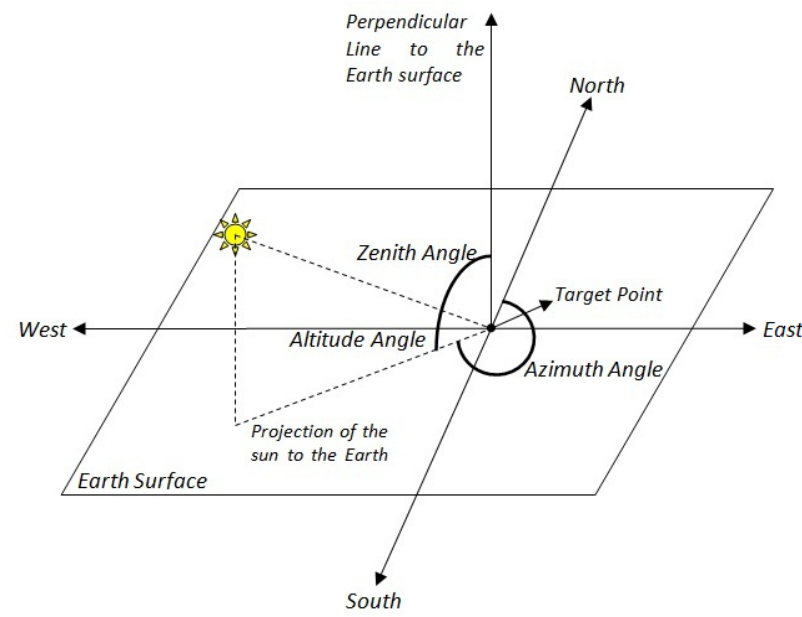

(a)

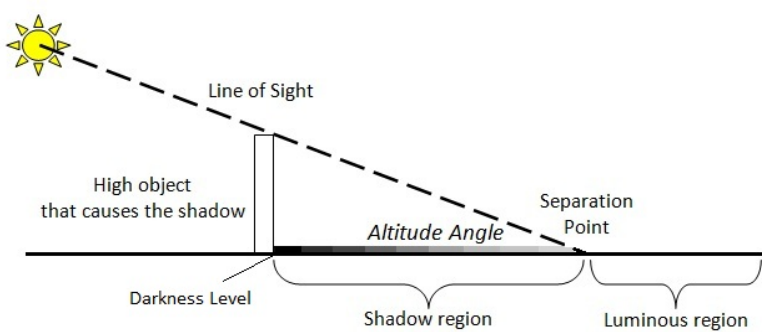

(b)

Figure 3. The presentation of sun angles (a) and the shadow region (b).

Each pixel in the elevation matrix is a target point individually. At the first step of the main loop in the LoS algorithm, a projection line of the sun over the earth's surface is determined by using the azimuth angle of the sun. This virtual projection line passes over the objects like building, vegetation etc. that could cause shadow on the target point by blocking the sun light. Furthermore, a limit for height parameter is calculated for the objects on the projection line by multiplying tangent of altitude angle and horizontal distance between the target pixel and the current pixel. If the height of the current pixel is higher than the limit, the target pixel is marked as shadow and the shadowiness level is calculated with respect to Equations 1 and 2. The results are saved into another matrix on the same column and row number of the target pixel as shown in Figure 5. This iteration is accomplished for all pixels in the elevation matrix in order to obtain the whole shadowy area. The shadowiness level can be computed as:

$$
\text { Shadowiness_Level }=k * \frac{\sqrt{l}}{h_{-} o}
$$

where, $k$ is the scaling factor, $l$ is the horizontal distance between the current pixel and the target pixel as given in Equation 2, $h_{-} o$ is the height of the object from LiDAR data that intersects the sun light and causes the shadow on the target pixel as shown in Figure 4. The horizontal distance can be computed as:

$$
l=\text { cellsize } * \sqrt{u^{2}+v^{2}}
$$

where $u$ and $v$ are the difference values on rows and columns respectively. 


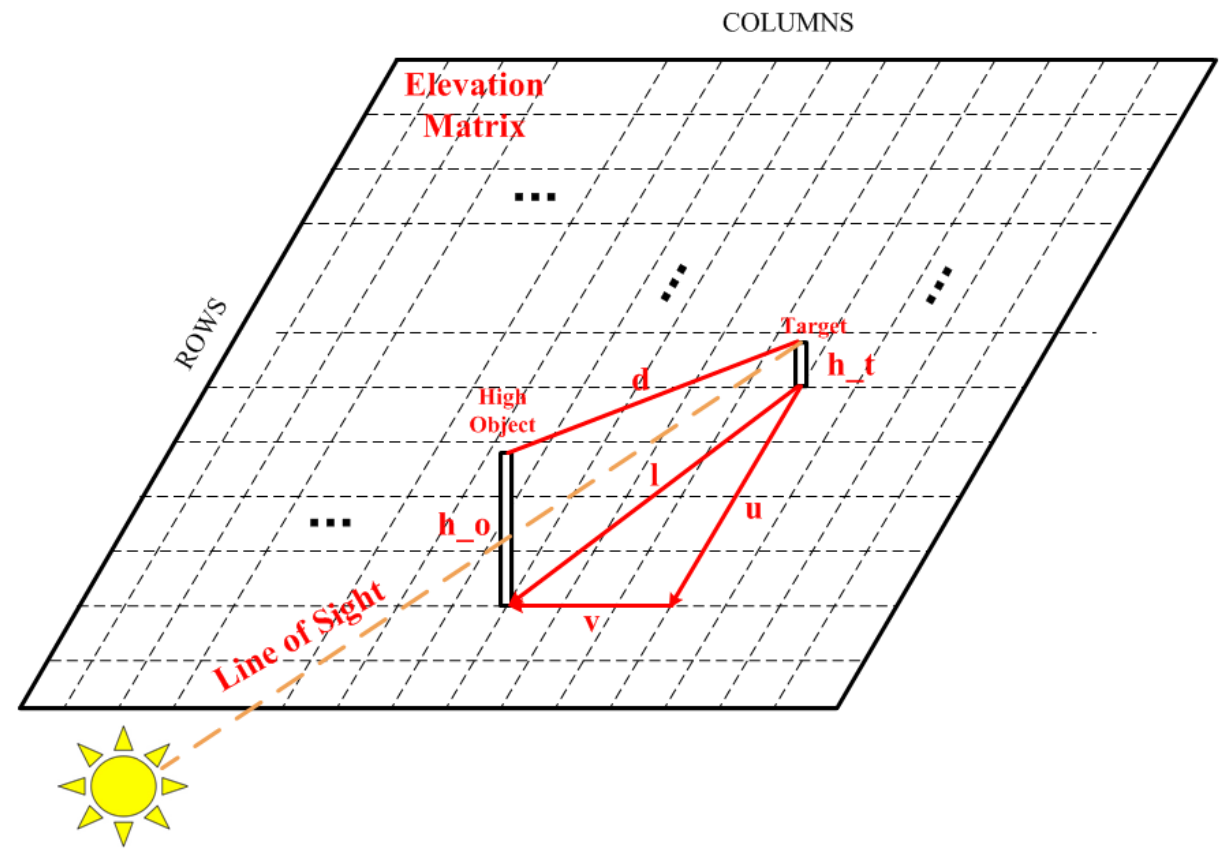

Figure 4. Definition of the parameters in Equations 1 and 2. A line of sight is plotted from the target object to the sun. If this LoS intersects with a high object as shown here, then the target is under shadow, and a shadowiness level is computed.

Additionally, the height of the target $\left(h_{-} t\right.$ ) was assumed as another important parameter for shadowiness level and a different equation was defined as shown in Equations 3 and 4. In Equation 3, the shadowiness level exponentially decreases with increasing distance as follows:

$$
\text { Shadowiness_Level }=\exp (t * d)
$$

where $t$ is another scaling factor that is different from $k, d$ is the distance between endpoints of the target point and current point as given below:

$$
\left.d=\sqrt{(\text { cellsize } * u)^{2}+(\text { cellsize } * v)^{2}+\left(h_{-} o-h_{-} t\right)^{2}}\right)
$$

and shown in Figure 4. Since there was not a significant difference between the outputs of the LoS algorithm with Equation 1 and 3, Equation 1 was used for the calculations in the rest of this paper.

Using these computations, shadow maps were generated as shown in the figure below. Figure 5.a is a usual map view of area 16 (flight line number 16 in LiDAR dataset) which was taken from Google Maps and the coordinates of the edge points were obtained by converting UTM coordinates from LiDAR dataset to latitude and longitude parameters as defined in WGS84 standard ${ }^{10}$. All shadow regions for area 16 are shown in Figure 5.b.

Furthermore, a building in the area 16, which is shown in Figure 5.c and 5.d, was chosen to analyze the effect of the shadow level differences on hyperspectral data. The shadow regions lay on different places in Figure 5.c and 5.d. The main reason of this difference is that Google may collect the map data from satellite at afternoon, although the hyperspectral dataset was collected in the morning, so, the position and angles of the sun are not same for these figures. 


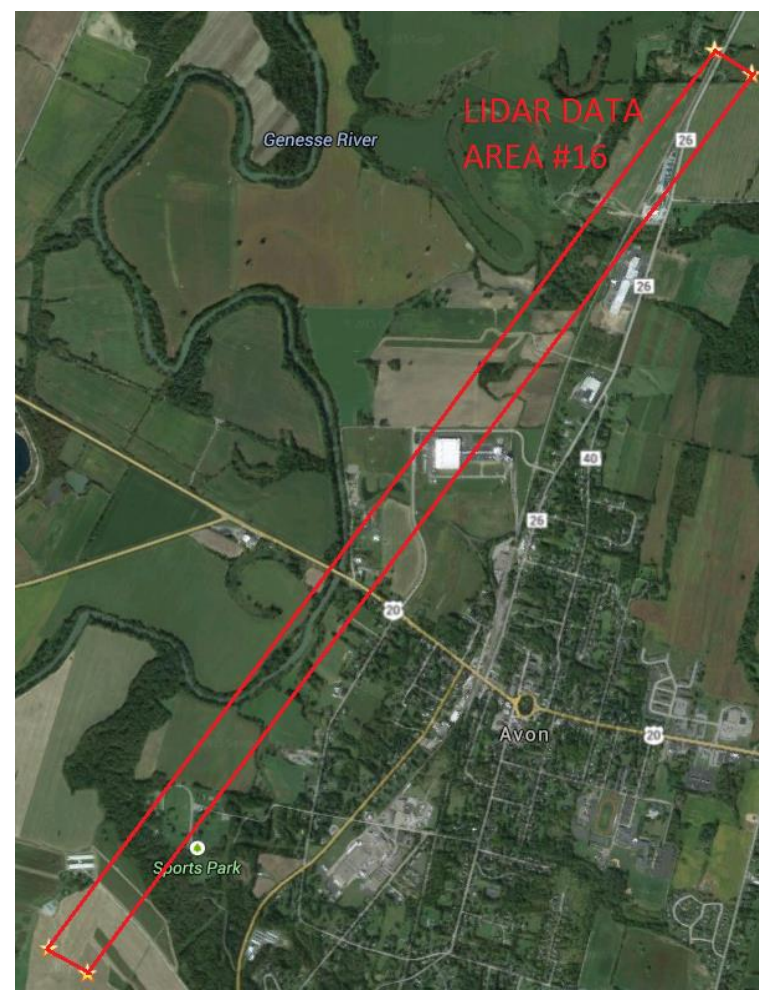

(a)

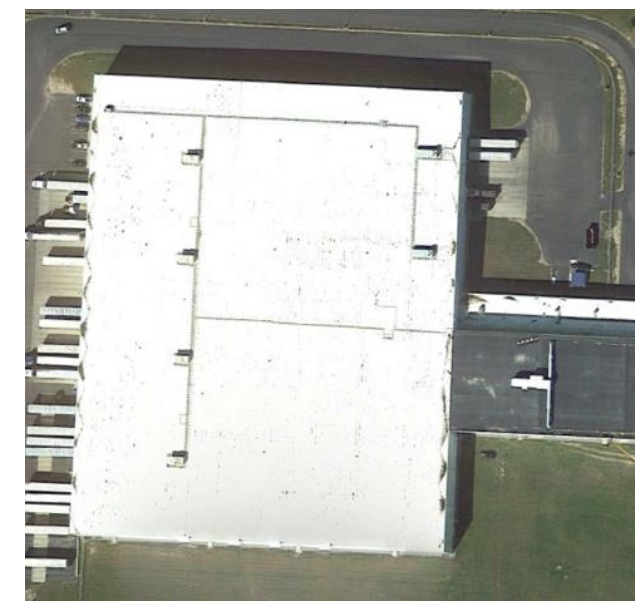

(c)

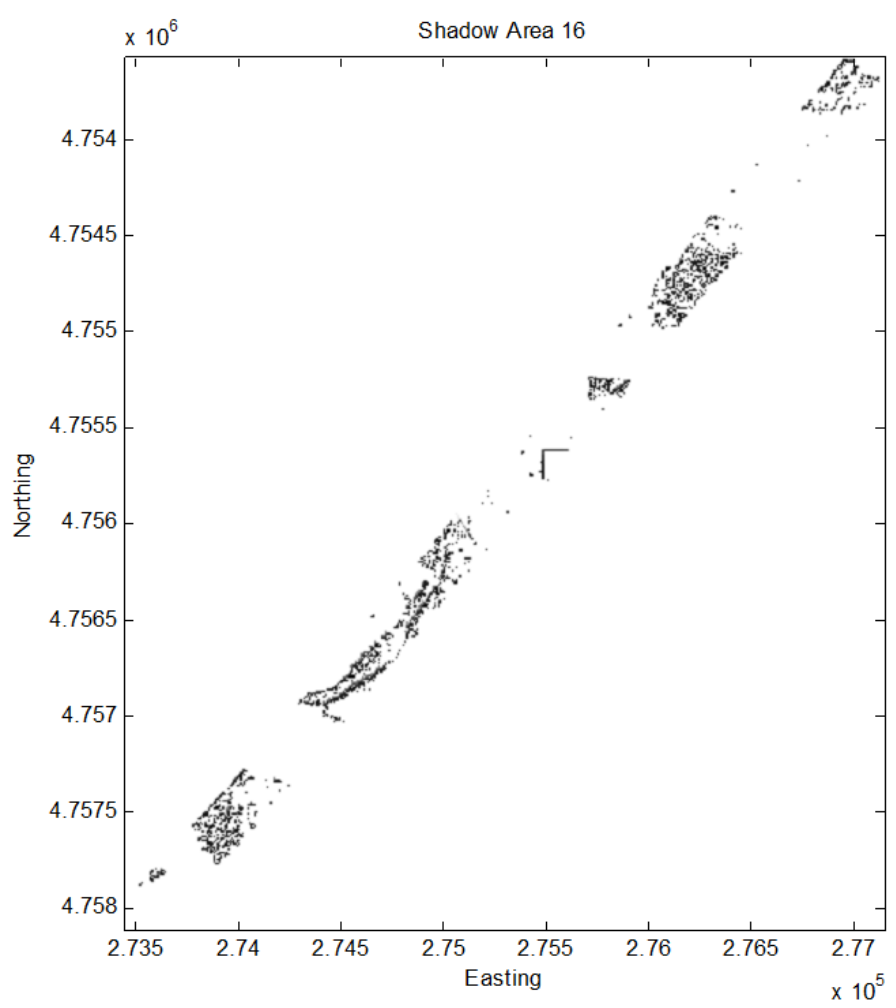

(b)

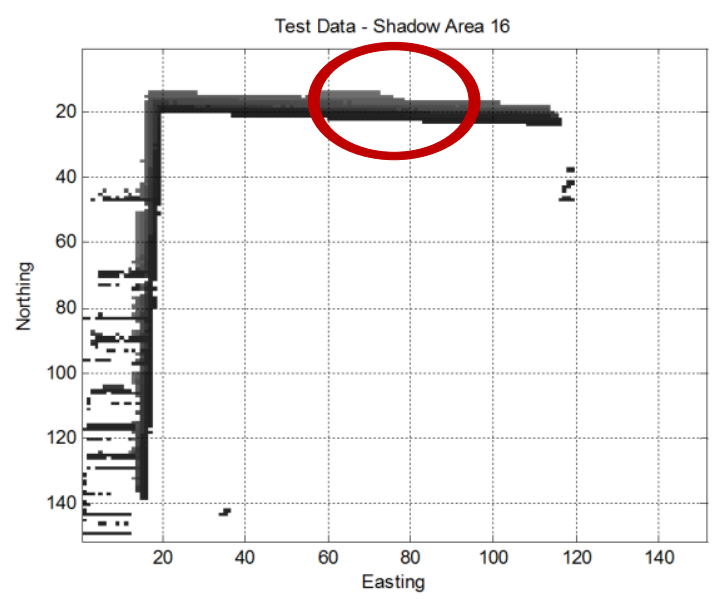

(d)

Figure 5. The output of LoS Algorithm, (a) Entire map of area 16 from Google Maps, (b) Calculated shadow regions for area 16, (c) map of a sample building in area 16 from Google Maps, (d) Calculated shadow regions for the sample building. 


\section{Analyzing the Effect of Shadowiness Difference on Hyperspectral Data}

In a hyperspectral scene, the reflectance values of shadow areas are suppressed according to luminous areas even if these areas are made of exactly same material like grass, sand, stone etc. Also, the shadowiness level difference may cause dissimilarity in the reflectance values of the neighbor shadow points. Some points are marked from Figure 5.c to investigate these theorems as shown in Figure 6.

In Figure 6, some sample points are selected from the edge of the sample building in Figure 5.c and d. to analyze the effect of the shadow and shadowiness difference on the reflectance values. The LoS algorithm determined that these sample points are shadow or not and assigned a specific shadowiness level to each point. The values of the sample points are inversely proportional with the intensity of the shadow such that the value of 10.44 specifies that the point is not shadow and the value of 1.32 specifies that the shadow intensity of this point is the highest among them. Also, LoS algorithm calculated the coordinates (easting and northing) of these points and saved them into another matrix. The reflectance value of a shadow point was found by matching the easting and northing values individually with $\pm 0.5 \mathrm{~m}$ tolerances which were obtained from LiDAR dataset with LoS algorithm and hyperspectral dataset.

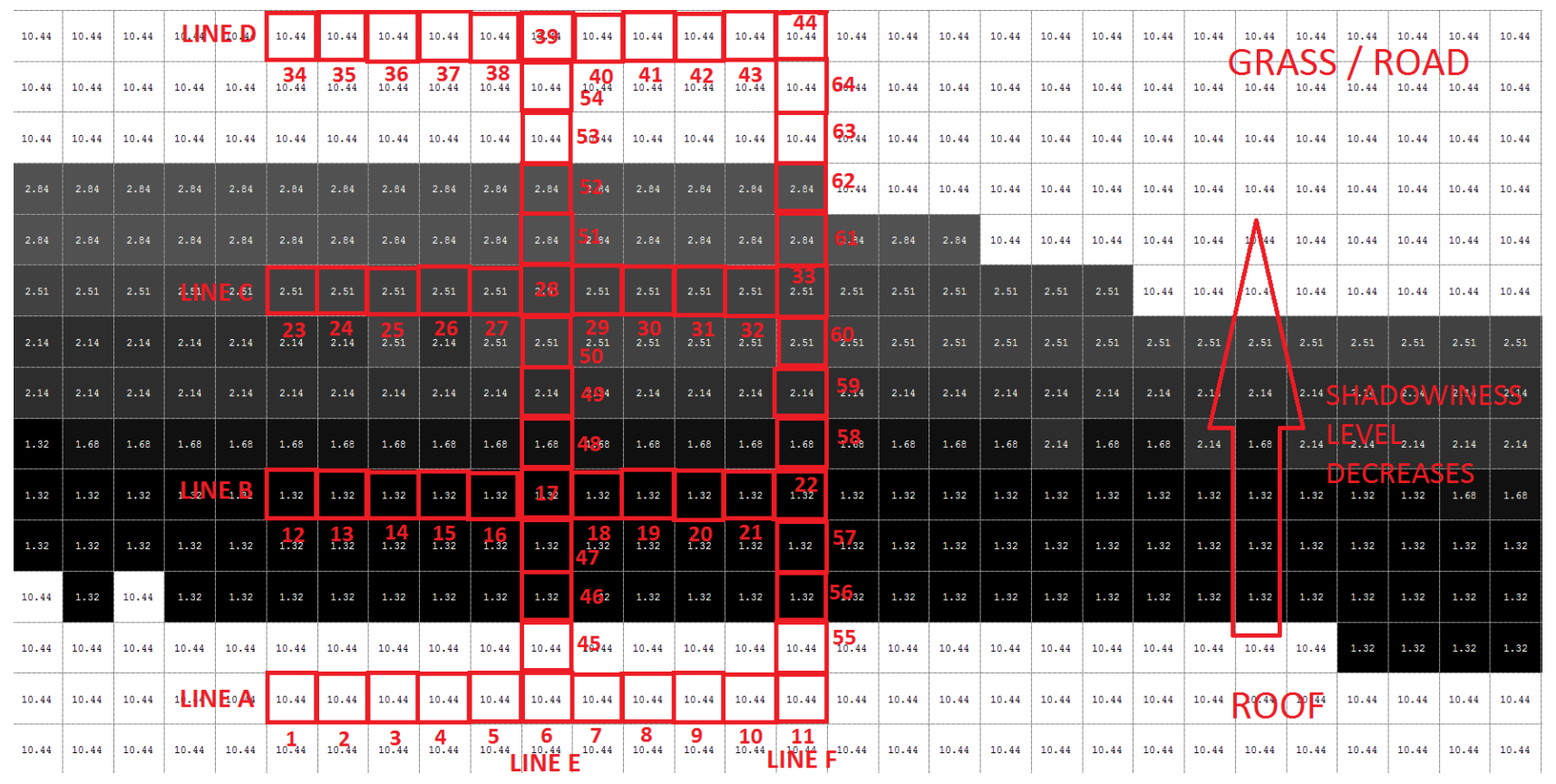

Figure 6. Hyperspectral data was plotted for these sample points which are taken from the edge of the sample building that is shown in red in Figure 5.d. Furthermore, in the figure, the shadowiness level for all points are shown as inverse proportionally in the each square.

In Figure 7, there are the reflectance plots for some selected points from Figure 6 which the point as numbered 6 exists on the roof and the point as numbered 59 exists on the grass. As shown in Figure 7.a, the reflectance values are not suppressed for any wavelength values as expected. Figure 7.b, c, d and e prove that the reflectance values of shadow areas are lower than the luminous areas' and the LoS algorithm marks the points as shadow correctly although the distance between two neighbor sample points is 1.278 (cellsize) \pm 0.5 meters.

On the other hand, in spite of the shadow intensities are same for the points 6 and 39, the reflectance plots are quite different as shown in Fig. 7. The main reason of this difference is that the raw materials of these points are different as a result of analyses of reflectance plots of the other points. 


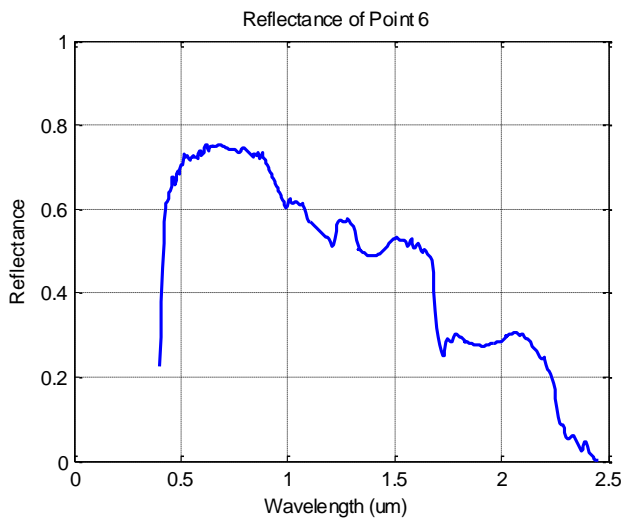

(a) Point 6, SL 10.44 (not shadow)

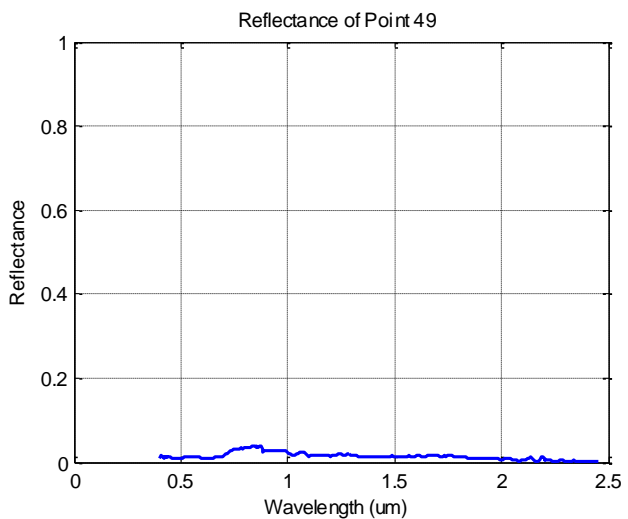

(c) Point 49, SL 2.14

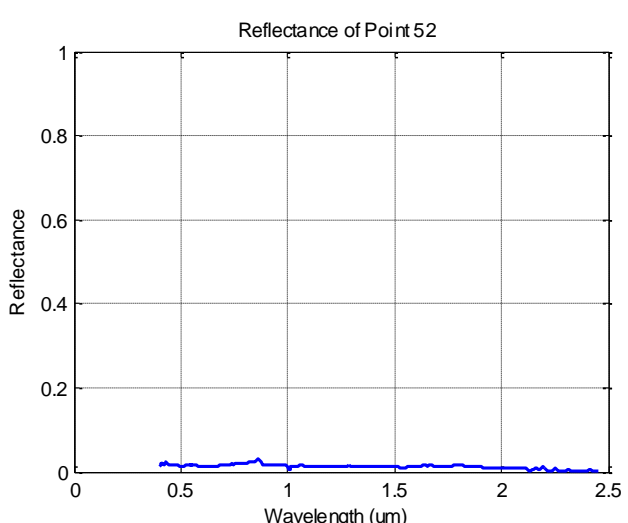

(e) Point 52, SL 2.84 (minimum intensity)

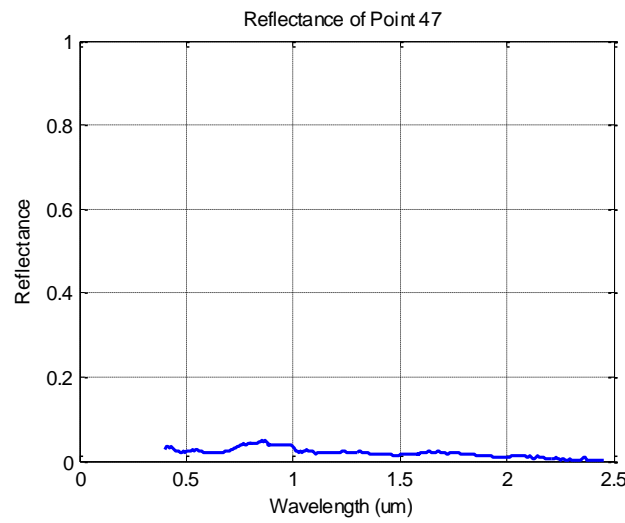

(b) Point 47, SL 1.32 (maximum intensity)

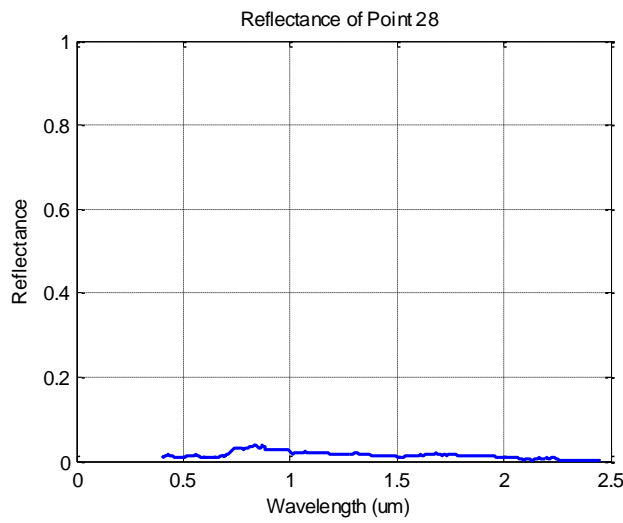

(d) Point 28, SL 2.51

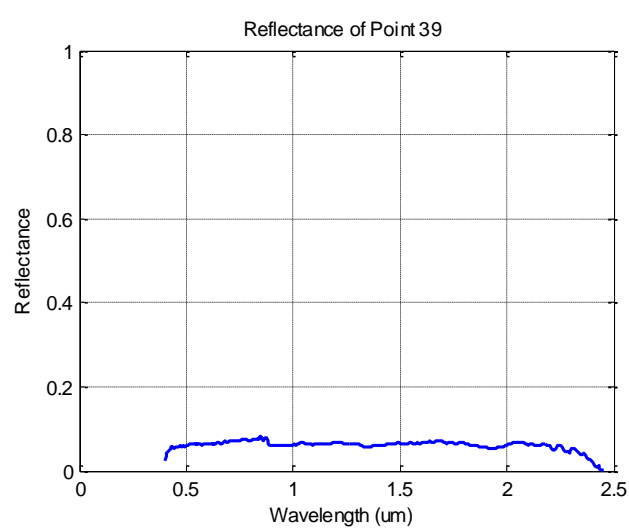

(f) Point 39, SL 10.44 (not shadow)

Figure 7. Hyperspectral data (reflectance) was plotted for 6 (a), 47 (b), 49 (c), 28 (d), 52 (e) and 39 (f) numbered points from Line E in the Figure 6. Shadowiness level (SL) of these points are 10.44 (not shadow), 1.32 (maximum intensity), 2.14, 2.51, 2.84 (minimum intensity) and 10.44 (not shadow) respectively.

\section{CONCLUSIONS}

This study aimed to,

- find the shadow areas and assign a shadowiness level by using LiDAR dataset with a simply, effective and specific Line of Sight algorithm which was based on Matlab interface, 
- verify the correctness of the LoS algorithm and analyze the effect of the shadow areas on the reflectance values for all wavelengths by using hyperspectral dataset,

- $\quad$ and finally, investigate the changes of the reflectance values with respect to the shadowiness level.

The developed LoS algorithm found the shadow areas, calculated the easting and northing coordinates, generated a shadowiness level for each pixel in the elevation matrix and saved all these information.

On the other hand, there was no ground truth data that labelled the shadow areas at the certain time and date. Hence, the hyperspectral dataset was used to verify the correctness of the LoS algorithm by comparing the reflectance of the neighbor points in Figure 6. The reflectance values of the all points in Fig. 6 were plotted and compared among all wavelengths. Even if the horizontal distance between the points are very short (a few meters), the reflectance of the points, which have been marked as shadow, are suppressed as shown in Fig. 7. The same procedure was applied to other areas, such as a forest, and the reflectance plots of shadowy areas were suppressed again. These samples and plots indicate that the LoS algorithm finds the shadow areas correctly.

Furthermore, the same plots of the sample points were analyzed to bring out the effect of the shadowiness difference on the reflectance value. Although the lowest reflectance values were expected for the shadowy areas where the intensity of the shadow was maximum and vice versa, the plots showed that there were not any significant difference on the reflectance values as shown in Fig. 7. Even, the reflectance values of high shadowiness level came out higher than low shadowiness level in the woodland areas. The main reason of this problem was that laser light may pass through between the leafs. This is one area that needs further investigation. While benefitting from vast application opportunities of using LiDAR data, there might be disadvantages and performance issues due to resolution of wide area, conversion of file formats.

\section{REFERENCES}

[1] Ientilucci, E. J., "Leveraging Lidar Data to Aid in Hyperspectral Image Target Detection in the Radiance Domain," Proc. of SPIE Vol. 8390 839007-1 (2012)

[2] Shimoni, M., Tolt, G., Perneel, C., Ahlberg, J., "Detection of Vehicles in Shadow Areas," Workshop Hyperspectral Image Signal Process Evol. Remote Sens. (Whispers), $20113^{\text {rd }}$ Workshop on (pp. 1-4) IEEE (2011, June)

[3] Shimoni, M., Tolt, G., Perneel, C., Ahlberg, J., "Detection Of Vehicles In Shadow Areas Using Combined Hyperspectral And Lidar Data, " Geoscience and Remote Sensing Symposium (IGARSS), IEEE, pp. 4427-4430 (2011, July)

[4] Guth, P. L., "Probabilistic Line-of-Sight with Lidar Point Clouds," ASPRS 2012 Annual Conference, California (19-23 March 2012)

[5] Tolt, G., Shimoni, M., Ahlberg, J., "A Shadow Detection Method For Remote Sensing Images Using VHR Hyperspectral and Lidar Data," Geoscience and Remote Sensing Symposium (IGARSS), IEEE, pp. 4423-4426 (2011, July)

[6] Giannandrea, A., Raqueno, N., Messinger, D. W., Faulring, J., Kerekes, J. P., van Aardt, J., Daniel, B. "The SHARE 2012 Data Campaign," Proc. of SPIE Vol. 8743, 87430F-1 (2013, May)

[7] SHARE 2012, "LiDAR," Rochester Institute of Technology, 23 March 2015 http://www.rit.edu/cos/share2012/lidar.php

[8] SHARE 2012, "SpecTIR Overview Project Report," Rochester Institute of Technology, 23 March 2015 http://www.rit.edu/cos/share2012/downloadarea.php

[9] SHARE 2012, "SpecTIR," Rochester Institute of Technology, 23 March 2015 http://www.rit.edu/cos/share2012/spectir.php

[10] Sandwell, D. T., "Reference Earth Model-WGS84," Scripps Institution of Oceanography (2002)

[11] Ientilucci, E. J., "SHARE 2012: Analysis of Illumination Differences on Targets in Hyperspectral Imagery," Proc. of SPIE Vol. 8743 87430I-1 (2013, May)

[12]Zhang, Q., Pauca, V. P., Plemmons, R. J., Nikic, D. D., "Detecting Objects under Shadows by Fusion of Hyperspectral and Lidar Data: A Physical Model Approach," Workshop Hyperspectral Image Signal Process Evol. Remote Sens. (Whispers) (2013)

[13] MathWorks, "Speeding Up MATLAB Applications," Whitepaper 91991v01 03/13 